\title{
Triple solutions of complementary Lidstone boundary value problems via fixed point theorems
}

\section{Patricia JY Wong*}

\section{"Correspondence:} ejywong@ntu.edu.sg School of Electrical and Electronic Engineering, Nanyang Technological University, 50 Nanyang Avenue, Singapore 639798, Singapore

\begin{abstract}
We consider the following complementary Lidstone boundary value problem:

$$
\begin{aligned}
& (-1)^{m} y^{(2 m+1)}(t)=F\left(t, y(t), y^{\prime}(t)\right), \quad t \in[0,1] \\
& y(0)=0, \quad y^{(2 k-1)}(0)=y^{(2 k-1)}(1)=0, \quad 1 \leq k \leq m .
\end{aligned}
$$

By using fixed point theorems of Leggett-Williams and Avery, we offer several criteria for the existence of three positive solutions of the boundary value problem. Examples are also included to illustrate the results obtained. We note that the nonlinear term $F$ depends on $y^{\prime}$ and this derivative dependence is seldom investigated in the literature and a new technique is required to tackle the problem.
\end{abstract}

MSC: $34 \mathrm{~B} 15 ; 34 \mathrm{~B} 18$

Keywords: positive solutions; complementary Lidstone boundary value problems; derivative-dependent nonlinearity; fixed point theorems

\section{Introduction}

In this paper we shall consider the complementary Lidstone boundary value problem

$$
\begin{aligned}
& (-1)^{m} y^{(2 m+1)}(t)=F\left(t, y(t), y^{\prime}(t)\right), \quad t \in[0,1], \\
& y(0)=0, \quad y^{(2 k-1)}(0)=y^{(2 k-1)}(1)=0, \quad 1 \leq k \leq m,
\end{aligned}
$$

where $m \geq 1$ and $F$ is continuous at least in the interior of the domain of interest. It is noted that the nonlinear term $F$ involves $y^{\prime}$, a derivative of the dependent variable. Most research papers on boundary value problems consider nonlinear terms that involve $y$ only, and derivative-dependent nonlinearities are seldom tackled as special techniques are required.

The complementary Lidstone interpolation and boundary value problems have been very recently introduced in [1], and drawn on by Agarwal et al. in $[2,3]$ where they consider an $(2 m+1)$ th order differential equation together with boundary data at the odd order derivatives

$$
y(0)=a_{0}, \quad y^{(2 k-1)}(0)=a_{k}, \quad y^{(2 k-1)}(1)=b_{k}, \quad 1 \leq k \leq m .
$$


The boundary conditions (1.2) are known as complementary Lidstone boundary conditions, they naturally complement the Lidstone boundary conditions [4-7] which involve even order derivatives. To be precise, the Lidstone boundary value problem comprises an $2 m$ th order differential equation and the Lidstone boundary conditions

$$
y^{(2 k)}(0)=a_{k}, \quad y^{(2 k)}(1)=b_{k}, \quad 0 \leq k \leq m-1 .
$$

There is a vast literature on Lidstone interpolation and boundary value problems. In fact, the Lidstone interpolation was first introduced by Lidstone [8] in 1929 and further characterized in the work of [9-16]. More recent research on Lidstone interpolation as well as Lidstone spline can be found in [1, 17-23]. Meanwhile, the Lidstone boundary value problems and several of its particular cases have been the subject matter of numerous investigations, see $[4,18,24-37]$ and the references cited therein. In most of these works the nonlinear terms considered do not involve derivatives of the dependent variable, only a handful of papers $[30,31,34,35]$ tackle nonlinear terms that involve even order derivatives. In the present work, our study of the complementary Lidstone boundary value problem (1.1) where $F$ depends on a derivative certainly extends and complements the rich literature on boundary value problems and notably on Lidstone boundary value problems. The literature on complementary Lidstone boundary value problems pales in comparison with that on Lidstone boundary value problems - after the first work [2] on complementary Lidstone boundary value problems, the recent paper [38] discusses the eigenvalue problem, while in [39] the existence of at least one or two positive solutions of the complementary Lidstone boundary value problem is derived by Leray-Schauder alternative and Krasnosel'skii's fixed point theorem in a cone.

In the present work, we shall establish the existence of at least three positive solutions using fixed point theorems of Leggett and Williams [40] as well as of Avery [41]. Estimates on the norms of these solutions will also be provided. Besides achieving new results, we also compare the results in terms of generality and illustrate the importance of the results through some examples. As remarked earlier, the presence of the derivative $y^{\prime}$ in the nonlinear term $F$ requires a special technique to tackle the problem.

The paper is organized as follows. Section 2 contains the necessary definitions and fixed point theorems. The existence criteria are developed and discussed in Section 3. Finally, examples are presented in Section 4 to illustrate the importance of the results obtained.

\section{Preliminaries}

In this section we shall state some necessary definitions, the relevant fixed point theorems and properties of certain Green's function. Let $B$ be a Banach space equipped with the norm $\|\cdot\|$.

Definition 2.1 Let $C(\subset B)$ be a nonempty closed convex set. We say that $C$ is a cone provided the following conditions are satisfied:

(a) If $x \in C$ and $\alpha \geq 0$, then $\alpha x \in C$;

(b) If $x \in C$ and $-x \in C$, then $x=0$.

Definition 2.2 Let $C(\subset B)$ be a cone. A map $\psi$ is a nonnegative continuous concave functional on $C$ if the following conditions are satisfied: 
(a) $\psi: C \rightarrow[0, \infty)$ is continuous;

(b) $\psi(t y+(1-t) z) \geq t \psi(y)+(1-t) \psi(z)$ for all $y, z \in C$ and $0 \leq t \leq 1$.

Definition 2.3 Let $C(\subset B)$ be a cone. A map $\beta$ is a nonnegative continuous convex functional on $C$ if the following conditions are satisfied:

(a) $\beta: C \rightarrow[0, \infty)$ is continuous;

(b) $\beta(t y+(1-t) z) \leq t \beta(y)+(1-t) \beta(z)$ for all $y, z \in C$ and $0 \leq t \leq 1$.

Let $\gamma, \beta$, $\Theta$ be nonnegative continuous convex functionals on $C$ and $\alpha$, $\psi$ be nonnegative continuous concave functionals on $C$. For nonnegative numbers $w_{i}, 1 \leq i \leq 3$, we shall introduce the following notations:

$$
\begin{aligned}
& C\left(w_{1}\right)=\left\{x \in C \mid\|x\|<w_{1}\right\}, \\
& C\left(\psi, w_{1}, w_{2}\right)=\left\{x \in C \mid \psi(x) \geq w_{1} \text { and }\|x\| \leq w_{2}\right\}, \\
& P\left(\gamma, w_{1}\right)=\left\{x \in C \mid \gamma(x)<w_{1}\right\}, \\
& P\left(\gamma, \alpha, w_{1}, w_{2}\right)=\left\{x \in C \mid \alpha(x) \geq w_{1} \text { and } \gamma(x) \leq w_{2}\right\}, \\
& Q\left(\gamma, \beta, w_{1}, w_{2}\right)=\left\{x \in C \mid \beta(x) \leq w_{1} \text { and } \gamma(x) \leq w_{2}\right\}, \\
& P\left(\gamma, \Theta, \alpha, w_{1}, w_{2}, w_{3}\right)=\left\{x \in C \mid \alpha(x) \geq w_{1}, \Theta(x) \leq w_{2} \text { and } \gamma(x) \leq w_{3}\right\}, \\
& Q\left(\gamma, \beta, \psi, w_{1}, w_{2}, w_{3}\right)=\left\{x \in C \mid \psi(x) \geq w_{1}, \beta(x) \leq w_{2} \text { and } \gamma(x) \leq w_{3}\right\} .
\end{aligned}
$$

The following fixed point theorems are our main tools, the first is usually called LeggettWilliams' fixed point theorem, and the second is known as the five-functional fixed point theorem.

Theorem 2.1 [40] Let $C(\subset B)$ be a cone, and $w_{4}>0$ be given. Assume that $\psi$ is a nonnegative continuous concave functional on $C$ such that $\psi(x) \leq\|x\|$ for all $x \in \bar{C}\left(w_{4}\right)$, and let $S: \bar{C}\left(w_{4}\right) \rightarrow \bar{C}\left(w_{4}\right)$ be a continuous and completely continuous operator. Suppose that there exist numbers $w_{1}, w_{2}, w_{3}$, where $0<w_{1}<w_{2}<w_{3} \leq w_{4}$, such that

(a) $\left\{x \in C\left(\psi, w_{2}, w_{3}\right) \mid \psi(x)>w_{2}\right\} \neq \emptyset$, and $\psi(S x)>w_{2}$ for all $x \in C\left(\psi, w_{2}, w_{3}\right)$;

(b) $\|S x\|<w_{1}$ for all $x \in \bar{C}\left(w_{1}\right)$;

(c) $\psi(S x)>w_{2}$ for all $x \in C\left(\psi, w_{2}, w_{4}\right)$ with $\|S x\|>w_{3}$.

Then $S$ has (at least) three fixed points $x_{1}, x_{2}$ and $x_{3}$ in $\bar{C}\left(w_{4}\right)$. Furthermore, we have

$$
\begin{aligned}
& x_{1} \in C\left(w_{1}\right), \quad x_{2} \in\left\{x \in C\left(\psi, w_{2}, w_{4}\right) \mid \psi(x)>w_{2}\right\} \quad \text { and } \\
& x_{3} \in \bar{C}\left(w_{4}\right) \backslash\left(C\left(\psi, w_{2}, w_{4}\right) \cup \bar{C}\left(w_{1}\right)\right) .
\end{aligned}
$$

Theorem 2.2 [41] Let $C(\subset B)$ be a cone. Assume that there exist positive numbers $w_{5}$, $M$, nonnegative continuous convex functionals $\gamma, \beta, \Theta$ on $C$, and nonnegative continuous concave functionals $\alpha, \psi$ on $C$, with

$$
\alpha(x) \leq \beta(x) \quad \text { and } \quad\|x\| \leq M \gamma(x)
$$

for all $x \in \bar{P}\left(\gamma, w_{5}\right)$. Let $S: \bar{P}\left(\gamma, w_{5}\right) \rightarrow \bar{P}\left(\gamma, w_{5}\right)$ be a continuous and completely continuous operator. Suppose that there exist nonnegative numbers $w_{i}, 1 \leq i \leq 4$, with $0<w_{2}<w_{3}$ such that 
(a) $\left\{x \in P\left(\gamma, \Theta, \alpha, w_{3}, w_{4}, w_{5}\right) \mid \alpha(x)>w_{3}\right\} \neq \emptyset$, and $\alpha(S x)>w_{3}$ for all $x \in P\left(\gamma, \Theta, \alpha, w_{3}, w_{4}, w_{5}\right)$;

(b) $\left\{x \in Q\left(\gamma, \beta, \psi, w_{1}, w_{2}, w_{5}\right) \mid \beta(x)<w_{2}\right\} \neq \emptyset$, and $\beta(S x)<w_{2}$ for all $x \in Q\left(\gamma, \beta, \psi, w_{1}, w_{2}, w_{5}\right)$;

(c) $\alpha(S x)>w_{3}$ for all $x \in P\left(\gamma, \alpha, w_{3}, w_{5}\right)$ with $\Theta(S x)>w_{4}$;

(d) $\beta(S x)<w_{2}$ for all $x \in Q\left(\gamma, \beta, w_{2}, w_{5}\right)$ with $\psi(S x)<w_{1}$.

Then $S$ has (at least) three fixed points $x_{1}, x_{2}$ and $x_{3}$ in $\bar{P}\left(\gamma, w_{5}\right)$. Furthermore, we have

$$
\beta\left(x_{1}\right)<w_{2}, \quad \alpha\left(x_{2}\right)>w_{3} \text { and } \beta\left(x_{3}\right)>w_{2} \quad \text { with } \alpha\left(x_{3}\right)<w_{3} .
$$

We also require the definition of an $L^{1}$-Carathéodory function.

Definition 2.4 [42] A function $P:[0,1] \times \mathbb{R}^{2} \rightarrow \mathbb{R}$ is an $L^{1}$-Carathéodory function if the following conditions hold:

(a) The map $t \rightarrow P(t, u)$ is measurable for all $u \in \mathbb{R}^{2}$.

(b) The map $u \rightarrow P(t, u)$ is continuous for almost all $t \in[0,1]$.

(c) For any $r>0$, there exists $\mu_{r} \in L^{1}[0,1]$ such that $|u| \leq r$ implies that $|P(t, u)| \leq \mu_{r}(t)$ for almost all $t \in[0,1]$.

To tackle the complementary Lidstone boundary value problem (1.1), let us review certain attributes of the Lidstone boundary value problem. Let $g_{m}(t, s)$ be the Green's function of the Lidstone boundary value problem

$$
\begin{aligned}
& x^{(2 m)}(t)=0, \quad t \in[0,1], \\
& x^{(2 k)}(0)=x^{(2 k)}(1)=0, \quad 0 \leq k \leq m-1 .
\end{aligned}
$$

The Green's function $g_{m}(t, s)$ can be expressed as $[4,5]$

$$
g_{m}(t, s)=\int_{0}^{1} g_{1}(t, u) g_{m-1}(u, s) d u, \quad m \geq 2,
$$

where

$$
g_{1}(t, s)= \begin{cases}t(s-1), & 0 \leq t \leq s \leq 1, \\ s(t-1), & 0 \leq s \leq t \leq 1 .\end{cases}
$$

Further, it is known that

$$
\left|g_{m}(t, s)\right|=(-1)^{m} g_{m}(t, s) \quad \text { and } \quad g_{m}(t, s)=g_{m}(s, t), \quad(t, s) \in(0,1) \times(0,1) \text {. }
$$

The following two lemmas give the upper and lower bounds of $\left|g_{m}(t, s)\right|$, they play an important role in subsequent development. We remark that the bounds in the two lemmas are sharper than those given in the literature $[4,5,35,37]$.

Lemma $2.1[38]$ For $(t, s) \in[0,1] \times[0,1]$, we have

$$
\left|g_{m}(t, s)\right| \leq \frac{1}{\pi^{2 m-1}} \sin \pi s .
$$


Lemma 2.2 [38] Let $\delta \in\left(0, \frac{1}{2}\right)$ be given. For $(t, s) \in[\delta, 1-\delta] \times[0,1]$, we have

$$
\left|g_{m}(t, s)\right| \geq \frac{2 \delta}{\pi^{2 m}} \sin \pi s
$$

\section{Triple positive solutions}

In this section, we shall use the fixed point theorems stated in Section 2 to obtain the existence of at least three positive solutions of the complementary Lidstone boundary value problem (1.1). By a positive solution $y$ of (1.1), we mean a nontrivial $y \in C[0,1]$ satisfying (1.1) and $y(t) \geq 0$ for $t \in[0,1]$.

To tackle (1.1), we first consider the initial value problem

$$
\begin{aligned}
& y^{\prime}(t)=x(t), \quad t \in[0,1], \\
& y(0)=0
\end{aligned}
$$

whose solution is simply

$$
y(t)=\int_{0}^{t} x(s) d s .
$$

Taking into account (3.1) and (3.2), the complementary Lidstone boundary value problem (1.1) reduces to the Lidstone boundary value problem

$$
\begin{aligned}
& (-1)^{m} x^{(2 m)}(t)=F\left(t, \int_{0}^{t} x(s) d s, x(t)\right), \quad t \in[0,1] \\
& x^{(2 k-2)}(0)=x^{(2 k-2)}(1)=0, \quad 1 \leq k \leq m .
\end{aligned}
$$

If (3.3) has a solution $x^{*}$, then by virtue of (3.2), the boundary value problem (1.1) has a solution given by

$$
y^{*}(t)=\int_{0}^{t} x^{*}(s) d s
$$

So the existence of a solution of the complementary Lidstone boundary value problem (1.1) follows from the existence of a solution of the Lidstone boundary value problem (3.3). It is clear from (3.4) that $\left\|y^{*}\right\| \leq\left\|x^{*}\right\|$; moreover if $x^{*}$ is positive, so is $y^{*}$. With the tools in Section 2 and a technique to handle the nonlinear term $F$, we shall study the boundary value problem (1.1) via (3.3).

Let the Banach space $B=C[0,1]$ be equipped with the norm $\|x\|=\sup _{t \in[0,1]}|x(t)|$ for $x \in B$. Define the operator $S: C[0,1] \rightarrow C[0,1]$ by

$$
\begin{aligned}
S x(t) & =\int_{0}^{1}(-1)^{m} g_{m}(t, s) F\left(s, \int_{0}^{s} x(\tau) d \tau, x(s)\right) d s \\
& =\int_{0}^{1}\left|g_{m}(t, s)\right| F\left(s, \int_{0}^{s} x(\tau) d \tau, x(s)\right) d s, \quad t \in[0,1],
\end{aligned}
$$

where $g_{m}(t, s)$ is the Green's function given in (2.4). A fixed point $x^{*}$ of the operator $S$ is clearly a solution of the boundary value problem (3.3), and as seen earlier $y^{*}(t)=\int_{0}^{t} x^{*}(s) d s$ is a solution of (1.1). 
For easy reference, we shall list the conditions that are needed later. In these conditions the sets $K$ and $\tilde{K}$ are defined by

$$
\begin{aligned}
\tilde{K} & =\{x \in B \mid x(t) \geq 0, t \in[0,1]\}, \\
K & =\{x \in \tilde{K} \mid x(t)>0 \text { on some subset of }[0,1] \text { of positive measure }\} . \\
\text { (C1) } F & :[0,1] \times \mathbb{R}^{2} \rightarrow \mathbb{R} \text { is an } L^{1} \text {-Carathéodory function. }
\end{aligned}
$$

(C2) We have

$$
\begin{aligned}
& F(t, u, v) \geq 0, \quad u, v \in \tilde{K} \text {, a.e. } t \in(0,1) \quad \text { and } \\
& F(t, u, v)>0, \quad u, v \in K \text {, a.e. } t \in(0,1) .
\end{aligned}
$$

(C3) There exist continuous functions $f, v, \mu$ with $f:[0, \infty) \times[0, \infty) \rightarrow[0, \infty)$ and $v, \mu$ : $(0,1) \rightarrow[0, \infty)$ such that

$$
\mu(t) f(u, v) \leq F(t, u, v) \leq v(t) f(u, v), \quad u, v \in \tilde{K} \text {, a.e. } t \in(0,1) .
$$

(C4) There exists a number $0<\theta \leq 1$ such that

$$
\mu(t) \geq \theta v(t), \quad \text { a.e. } t \in(0,1) .
$$

If (C2) and (C3) hold, then it follows from (3.5) that for $x \in \tilde{K}$ and $t \in[0,1]$,

$$
\begin{aligned}
0 & \leq \int_{0}^{1}\left|g_{m}(t, s)\right| \mu(s) f\left(\int_{0}^{s} x(\tau) d \tau, x(s)\right) d s \leq S x(t) \\
& \leq \int_{0}^{1}\left|g_{m}(t, s)\right| v(s) f\left(\int_{0}^{s} x(\tau) d \tau, x(s)\right) d s .
\end{aligned}
$$

Let $\delta \in\left(0, \frac{1}{2}\right)$ be fixed. We define a cone $C$ in $B$ as

$$
C=\left\{x \in B \mid x(t) \geq 0 \text { for } t \in[0,1] \text {, and } \min _{t \in[\delta, 1-\delta]} x(t) \geq \frac{2 \delta \theta}{\pi}\|x\|\right\},
$$

where $\theta$ is given in (C4). Clearly, we have $C \subseteq \tilde{K}$.

Lemma 3.1 Let (C1)-(C4) hold. Then the operator $S$ defined in (3.5) is continuous and completely continuous, and $S$ maps $C$ into $C$.

Proof From (2.4) we have $g_{m}(t, s) \in C[0,1] \subseteq L^{\infty}[0,1], t \in[0,1]$ and the map $t \rightarrow g_{m}(t, s)$ is continuous from $[0,1]$ to $C[0,1]$. This together with $F:[0,1] \times \mathbb{R}^{2} \rightarrow \mathbb{R}$ is an $L^{1}$ Carathéodory function ensures (as in [42, Theorem 4.2.2]) that $S$ is continuous and completely continuous.

Let $x \in C$. From (3.7) we have $S x(t) \geq 0$ for $t \in[0,1]$. Next, using (3.7) and Lemma 2.1 gives for $t \in[0,1]$,

$$
\begin{aligned}
S x(t) & \leq \int_{0}^{1}\left|g_{m}(t, s)\right| v(s) f\left(\int_{0}^{s} x(\tau) d \tau, x(s)\right) d s \\
& \leq \frac{1}{\pi^{2 m-1}} \int_{0}^{1} v(s) f\left(\int_{0}^{s} x(\tau) d \tau, x(s)\right) \sin \pi s d s .
\end{aligned}
$$


Hence, we have

$$
\|S x\| \leq \frac{1}{\pi^{2 m-1}} \int_{0}^{1} \nu(s) f\left(\int_{0}^{s} x(\tau) d \tau, x(s)\right) \sin \pi s d s .
$$

Now, employing (3.7), Lemma 2.2, (C4) and (3.10), we find for $t \in[\delta, 1-\delta]$,

$$
\begin{aligned}
S x(t) & \geq \int_{0}^{1}\left|g_{m}(t, s)\right| \mu(s) f\left(\int_{0}^{s} x(\tau) d \tau, x(s)\right) d s \\
& \geq \frac{2 \delta}{\pi^{2 m}} \int_{0}^{1} \mu(s) f\left(\int_{0}^{s} x(\tau) d \tau, x(s)\right) \sin \pi s d s \\
& \geq \frac{2 \delta}{\pi^{2 m}} \int_{0}^{1} \theta v(s) f\left(\int_{0}^{s} x(\tau) d \tau, x(s)\right) \sin \pi s d s \\
& \geq \frac{2 \delta \theta}{\pi}\|S x\| .
\end{aligned}
$$

This leads to

$$
\min _{t \in[\delta, 1-\delta]} S x(t) \geq \frac{2 \delta \theta}{\pi}\|S x\|
$$

We have shown that $S x \in C$.

For subsequent results, we define the following constants for fixed $\delta \in\left(0, \frac{1}{2}\right)$ and $\tau_{1}, \tau_{2}, \tau_{3}, \tau_{4} \in[0,1]:$

$$
\left\{\begin{array}{l}
q=\frac{1}{\pi^{2 m-1}} \int_{0}^{1} v(s) \sin \pi s d s, \\
r=\min _{t \in[\delta, 1-\delta]} \int_{\frac{1}{2}}^{1-\delta}\left|g_{m}(t, s)\right| \mu(s) d s \\
p_{1}=\min _{t \in\left[\tau_{2}, \tau_{3}\right]} \int_{\frac{1}{2}}^{\tau_{3}}\left|g_{m}(t, s)\right| \mu(s) d s \\
p_{2}=\frac{1}{\pi^{2 m-1}} \int_{\tau_{1}}^{\tau_{4}} v(s) \sin \pi s d s, \\
p_{3}=\frac{1}{\pi^{2 m-1}} \int_{0}^{\tau_{1}} v(s) \sin \pi s d s+\frac{1}{\pi^{2 m-1}} \int_{\tau_{4}}^{1} v(s) \sin \pi s d s .
\end{array}\right.
$$

\section{Lemma 3.2 Let (C1)-(C4) hold, and assume}

(C5) the function $v(s) \sin \pi s>0$ on a subset of $[0,1]$ of positive measure.

Suppose that there exists a number $d>0$ such that for $u, v \in[0, d]$,

$$
f(u, v)<\frac{d}{q}
$$

Then

$$
S(\bar{C}(d)) \subseteq C(d) \subset \bar{C}(d)
$$

Proof Let $x \in \bar{C}(d)$. So $\|x\| \leq d$, which implies immediately that

$$
\int_{0}^{s} x(\tau) d \tau \leq d \quad \text { and } \quad x(s) \leq d, \quad s \in[0,1] .
$$


Then, using (3.9), (C5) and (3.12), we find for $t \in[0,1]$,

$$
\begin{aligned}
|S x(t)| & \leq \frac{1}{\pi^{2 m-1}} \int_{0}^{1} v(s) f\left(\int_{0}^{s} x(\tau) d \tau, x(s)\right) \sin \pi s d s \\
& <\frac{1}{\pi^{2 m-1}} \int_{0}^{1} v(s) \sin \pi s d s \cdot \frac{d}{q} \\
& =q \cdot \frac{d}{q}=d .
\end{aligned}
$$

This implies $\|S x\|<d$. Together with the fact that $S x \in C$ (Lemma 3.1), we have shown that $S x \in C(d)$. Conclusion (3.13) is now immediate.

Using a similar argument as Lemma 3.2, we have the following lemma.

Lemma 3.3 Let (C1)-(C4) hold. Suppose that there exists a number $d>0$ such that for $u, v \in[0, d]$,

$$
f(u, v) \leq \frac{d}{q} .
$$

Then

$$
S(\bar{C}(d)) \subseteq \bar{C}(d)
$$

We are now ready to establish the existence of three positive solutions for the complementary Lidstone boundary value problem (1.1). The first result below uses LeggettWilliams' fixed point theorem (Theorem 2.1).

Theorem 3.1 Let $\delta \in\left(0, \frac{1}{2}\right)$ be fixed. Let (C1)-(C5) hold, and assume

(C6) for each $t \in[\delta, 1-\delta]$, the function $\left|g_{m}(t, s)\right| \mu(s)>0$ on a subset of $\left[\frac{1}{2}, 1-\delta\right]$ of positive measure.

Suppose that there exist numbers $w_{1}, w_{2}, w_{3}$ with

$$
0<w_{1}<w_{2}\left(\frac{1}{2}-\delta\right)<w_{2}<\frac{\pi w_{2}}{2 \delta \theta} \leq w_{3}
$$

such that the following hold:

(P) $f(u, v)<\frac{w_{1}}{q}$ for $u, v \in\left[0, w_{1}\right]$;

(Q) one of the following holds:

(Q1) $\limsup _{u \rightarrow \infty, v \rightarrow \infty} \frac{f(u, v)}{u}<\frac{1}{q}$ or $\limsup _{u \rightarrow \infty, v \rightarrow \infty} \frac{f(u, v)}{v}<\frac{1}{q}$;

(Q2) there exists a number $d\left(\geq w_{3}\right)$ such that $f(u, v) \leq \frac{d}{q}$ for $u, v \in[0, d]$;

(R) $f(u, v)>\frac{w_{2}}{r}$ for $u \in\left[w_{2}\left(\frac{1}{2}-\delta\right), w_{3}\left(\frac{1}{2}-\delta\right)\right]$ and $v \in\left[w_{2}, w_{3}\right]$.

Then we have the following conclusions:

(a) The Lidstone boundary value problem (3.3) has (at least) three positive solutions $x_{1}, x_{2}, x_{3} \in C$ (where $C$ is defined in (3.8)) such that

$$
\left\{\begin{array}{l}
\left\|x_{1}\right\|<w_{1} ; \\
x_{2}(t)>w_{2}, \quad t \in[\delta, 1-\delta] ; \\
\left\|x_{3}\right\|>w_{1} \text { and } \min _{t \in[\delta, 1-\delta]} x_{3}(t)<w_{2} .
\end{array}\right.
$$


(b) The complementary Lidstone boundary value problem (1.1) has (at least) three positive solutions $y_{1}, y_{2}, y_{3}$ such that for $i=1,2,3$,

$$
\left\{\begin{array}{l}
y_{i}(t)=\int_{0}^{t} x_{i}(s) d s, \quad t \in[0,1] \\
\left\|y_{i}\right\| \leq\left\|x_{i}\right\| ; \quad y_{i}(t) \geq \frac{2 \delta \theta}{\pi}\left\|x_{i}\right\|(t-\delta), \quad t \in[\delta, 1-\delta]
\end{array}\right.
$$

(where $x_{i}^{\prime}$ s are those in conclusion (a)). We further have

$$
\left\{\begin{array}{l}
\left\|y_{1}\right\|<w_{1} \\
y_{2}(t)>w_{2}(t-\delta), \quad t \in[\delta, 1-\delta] \\
y_{3}(t)>\frac{2 \delta \theta}{\pi} w_{1}(t-\delta), \quad t \in[\delta, 1-\delta]
\end{array}\right.
$$

Proof We shall employ Theorem 2.1 with the cone $C$ defined in (3.8). First, we shall prove that condition $(\mathrm{Q})$ implies the existence of a number $w_{4}$, where $w_{4} \geq w_{3}$, such that

$$
S\left(\bar{C}\left(w_{4}\right)\right) \subseteq \bar{C}\left(w_{4}\right)
$$

Suppose that (Q2) holds. Then by Lemma 3.3 we immediately have (3.17) where we pick $w_{4}=d$. Suppose now that $\limsup _{u \rightarrow \infty, v \rightarrow \infty} \frac{f(u, v)}{u}<\frac{1}{q}$ of (Q1) is satisfied. Then there exist $N>0$ and $\epsilon<\frac{1}{q}$ such that

$$
\frac{f(u, v)}{u}<\epsilon, \quad u, v>N
$$

Let

$$
L=\max _{u, v \in[0, N]} f(u, v) .
$$

Noting (3.18), it is then clear that for $u, v \geq 0$,

$$
f(u, v) \leq L+\epsilon u
$$

Now, pick the number $w_{4}$ so that

$$
w_{4}>\max \left\{w_{3}, L\left(\frac{1}{q}-\epsilon\right)^{-1}\right\} \text {. }
$$

Let $x \in \bar{C}\left(w_{4}\right)$. Using (3.9), (3.19) and (3.20) yields for $t \in[0,1]$,

$$
\begin{aligned}
|S x(t)| & \leq \frac{1}{\pi^{2 m-1}} \int_{0}^{1} v(s) f\left(\int_{0}^{s} x(\tau) d \tau, x(s)\right) \sin \pi s d s \\
& \leq \frac{1}{\pi^{2 m-1}} \int_{0}^{1} v(s)\left(L+\epsilon \int_{0}^{s} x(\tau) d \tau\right) \sin \pi s d s \\
& \leq \frac{1}{\pi^{2 m-1}} \int_{0}^{1} v(s)\left(L+\epsilon w_{4}\right) \sin \pi s d s \\
& =q\left(L+\epsilon w_{4}\right) \\
& <q\left[w_{4}\left(\frac{1}{q}-\epsilon\right)+\epsilon w_{4}\right]=w_{4} .
\end{aligned}
$$


Hence, $\|S x\|<w_{4}$ and so $S x \in C\left(w_{4}\right) \subset \bar{C}\left(w_{4}\right)$. Thus, (3.17) follows immediately. Note that the argument is similar if we assume that $\lim _{\sup _{u \rightarrow \infty, v \rightarrow \infty}} \frac{f(u, v)}{v}<\frac{1}{q}$ of (Q1) is satisfied.

Let $\psi: C \rightarrow[0, \infty)$ be defined by

$$
\psi(x)=\min _{t \in[\delta, 1-\delta]} x(t) .
$$

Clearly, $\psi$ is a nonnegative continuous concave functional on $C$ and $\psi(x) \leq\|x\|$ for all $x \in C$.

We shall verify that condition (a) of Theorem 2.1 is satisfied. It is obvious that

$$
x(t)=\frac{w_{2}+w_{3}}{2} \in\left\{x \in C\left(\psi, w_{2}, w_{3}\right) \mid \psi(x)>w_{2}\right\}
$$

and so $\left\{x \in C\left(\psi, w_{2}, w_{3}\right) \mid \psi(x)>w_{2}\right\} \neq \emptyset$. Next, let $x \in C\left(\psi, w_{2}, w_{3}\right)$. Then $\psi(x) \geq w_{2}$ and $\|x\| \leq w_{3}$ which imply

$$
x(s) \in\left[w_{2}, w_{3}\right], \quad s \in[\delta, 1-\delta] \quad \text { and } \quad \int_{\delta}^{\frac{1}{2}} x(\tau) d \tau \in\left[w_{2}\left(\frac{1}{2}-\delta\right), w_{3}\left(\frac{1}{2}-\delta\right)\right] .
$$

Using (3.7), (3.21), (C6) and (R), it follows that

$$
\begin{aligned}
\psi(S x) & =\min _{t \in[\delta, 1-\delta]} S x(t) \\
& \geq \min _{t \in[\delta, 1-\delta]} \int_{0}^{1}\left|g_{m}(t, s)\right| \mu(s) f\left(\int_{0}^{s} x(\tau) d \tau, x(s)\right) d s \\
& \geq \min _{t \in[\delta, 1-\delta]} \int_{\frac{1}{2}}^{1-\delta}\left|g_{m}(t, s)\right| \mu(s) f\left(\int_{\delta}^{\frac{1}{2}} x(\tau) d \tau, x(s)\right) d s \\
& >\min _{t \in[\delta, 1-\delta]} \int_{\frac{1}{2}}^{1-\delta}\left|g_{m}(t, s)\right| \mu(s) d s \cdot \frac{w_{2}}{r} \\
& =r \cdot \frac{w_{2}}{r}=w_{2} .
\end{aligned}
$$

Therefore, we have shown that $\psi(S x)>w_{2}$ for all $x \in C\left(\psi, w_{2}, w_{3}\right)$.

Next, by condition (P) and Lemma 3.2 (with $d=w_{1}$ ), we have $S\left(\bar{C}\left(w_{1}\right)\right) \subseteq C\left(w_{1}\right)$. Hence, condition (b) of Theorem 2.1 is fulfilled.

Finally, we shall show that condition (c) of Theorem 2.1 holds. Let $x \in C\left(\psi, w_{2}, w_{4}\right)$ with $\|S x\|>w_{3}$. Using (3.7), Lemma 2.2, (C4), (3.10) and the inequality $\frac{\pi w_{2}}{2 \delta \theta} \leq w_{3}$, we find

$$
\begin{aligned}
\psi(S x) & \geq \min _{t \in[\delta, 1-\delta]} \int_{0}^{1}\left|g_{m}(t, s)\right| \mu(s) f\left(\int_{0}^{s} x(\tau) d \tau, x(s)\right) d s \\
& \geq \frac{2 \delta}{\pi^{2 m}} \int_{0}^{1} \mu(s) f\left(\int_{0}^{s} x(\tau) d \tau, x(s)\right) \sin \pi s d s \\
& \geq \frac{2 \delta}{\pi^{2 m}} \int_{0}^{1} \theta v(s) f\left(\int_{0}^{s} x(\tau) d \tau, x(s)\right) \sin \pi s d s \\
& \geq \frac{2 \delta \theta}{\pi}\|S x\| \\
& >\frac{2 \delta \theta}{\pi} w_{3} \geq w_{2} .
\end{aligned}
$$

Hence, we have proved that $\psi(S x)>w_{2}$ for all $x \in C\left(\psi, w_{2}, w_{4}\right)$ with $\|S x\|>w_{3}$. 
It now follows from Theorem 2.1 that the Lidstone boundary value problem (3.3) has (at least) three positive solutions $x_{1}, x_{2}, x_{3} \in \bar{C}\left(w_{4}\right)$ satisfying (2.1). It is easy to see that here (2.1) reduces to (3.14). This completes the proof of conclusion (a).

Finally, it is observed from (3.4) that the complementary Lidstone boundary value problem (1.1) has (at least) three positive solutions $y_{1}, y_{2}, y_{3}$ such that for $i=1,2,3$,

$$
y_{i}(t)=\int_{0}^{t} x_{i}(s) d s, \quad t \in[0,1] \quad \text { and } \quad\left\|y_{i}\right\| \leq\left\|x_{i}\right\| .
$$

Moreover, since $x_{i} \in C$, we get for $t \in[\delta, 1-\delta]$,

$$
y_{i}(t)=\int_{0}^{t} x_{i}(s) d s \geq \int_{\delta}^{t} x_{i}(s) d s \geq \int_{\delta}^{t} \frac{2 \delta \theta}{\pi}\left\|x_{i}\right\| d s=\frac{2 \delta \theta}{\pi}\left\|x_{i}\right\|(t-\delta) .
$$

Combining (3.22) and (3.23) gives (3.15) immediately.

Further, since $x_{2}(t)>w_{2}$ for $t \in[\delta, 1-\delta]$, we have for $t \in[\delta, 1-\delta]$,

$$
y_{2}(t)=\int_{0}^{t} x_{2}(s) d s>\int_{\delta}^{t} x_{2}(s) d s>\int_{\delta}^{t} w_{2} d s=w_{2}(t-\delta) .
$$

Hence, noting (3.14), (3.15) and (3.24), we get (3.16). This completes the proof of conclu$\operatorname{sion}(\mathrm{b})$.

We shall now employ the five-functional fixed point theorem (Theorem 2.2) to give other existence criteria. In applying Theorem 2.2 it is possible to choose the functionals and constants in different ways, indeed we shall do so and derive two results. Our first result below turns out to be a generalization of Theorem 3.1.

Theorem 3.2 Let $\delta \in\left(0, \frac{1}{2}\right)$ be fixed. Let $(\mathrm{C} 1)-(\mathrm{C} 4)$ hold. Assume that there exist numbers $\tau_{j}, 1 \leq j \leq 4$, with

$$
0 \leq \tau_{1} \leq \delta \leq \tau_{2}<\frac{1}{2}<\tau_{3} \leq 1-\delta \leq \tau_{4} \leq 1
$$

such that

(C7) for each $t \in\left[\tau_{2}, \tau_{3}\right]$, the function $\left|g_{m}(t, s)\right| \mu(s)>0$ on a subset of $\left[\frac{1}{2}, \tau_{3}\right]$ of positive measure;

(C8) the function $v(s) \sin \pi s>0$ on a subset of $\left[\tau_{1}, \tau_{4}\right]$ of positive measure.

Suppose that there exist numbers $w_{i}, 2 \leq i \leq 5$, with

$$
\left\{\begin{array}{l}
0<w_{2}<w_{3}<\frac{\pi w_{3}}{28 \theta} \leq w_{4} \leq w_{5}<\frac{q w_{2}}{p_{3}} \\
\tau_{1} w_{5}+\left(\tau_{4}-\tau_{1}\right) w_{2}<w_{3}\left(\frac{1}{2}-\tau_{2}\right)
\end{array}\right.
$$

such that the following hold:

(P) $f(u, v)<\frac{1}{p_{2}}\left(w_{2}-\frac{w_{5} p_{3}}{q}\right)$ for $u \in\left[0, \tau_{1} w_{5}+\left(\tau_{4}-\tau_{1}\right) w_{2}\right]$ and $v \in\left[0, w_{2}\right]$;

(Q) $f(u, v) \leq \frac{w_{5}}{q}$ for $u, v \in\left[0, w_{5}\right]$;

(R) $f(u, v)>\frac{w_{3}}{p_{1}}$ for $u \in\left[w_{3}\left(\frac{1}{2}-\tau_{2}\right), w_{4}\left(\frac{1}{2}-\tau_{2}\right)\right]$ and $v \in\left[w_{3}, w_{4}\right]$.

Then we have the following conclusions: 
(a) The Lidstone boundary value problem (3.3) has (at least) three positive solutions $x_{1}, x_{2}, x_{3} \in \bar{C}\left(w_{5}\right)$ (where $C$ is defined in (3.8)) such that

$$
\left\{\begin{array}{l}
x_{1}(t)<w_{2}, \quad t \in\left[\tau_{1}, \tau_{4}\right] \\
x_{2}(t)>w_{3}, \quad t \in\left[\tau_{2}, \tau_{3}\right] ; \\
\max _{t \in\left[\tau_{1}, \tau_{4}\right]} x_{3}(t)>w_{2} \text { and } \min _{t \in\left[\tau_{2}, \tau_{3}\right]} x_{3}(t)<w_{3} .
\end{array}\right.
$$

(b) The complementary Lidstone boundary value problem (1.1) has (at least) three positive solutions $y_{1}, y_{2}, y_{3}$ such that (3.15) holds for $i=1,2,3$. We further have

$$
\left\{\begin{array}{l}
y_{1}(t)<\tau_{1} \max _{s \in\left[0, \tau_{1}\right]} x_{1}(s)+\left(\tau_{4}-\tau_{1}\right) w_{2}, \quad t \in\left[\tau_{1}, \tau_{4}\right] \\
y_{2}(t)>w_{3}\left(t-\tau_{2}\right), \quad t \in\left[\tau_{2}, \tau_{3}\right] ; \\
y_{3}(t)>\frac{2 \delta \theta}{\pi} w_{2}(t-\delta), \quad t \in[\delta, 1-\delta] .
\end{array}\right.
$$

Proof We shall apply Theorem 2.2 with the cone $C$ defined in (3.8). We define the following five functionals on the cone $C$ :

$$
\left\{\begin{array}{l}
\gamma(x)=\|x\| \\
\psi(x)=\min _{t \in[\delta, 1-\delta]} x(t) \\
\beta(x)=\Theta(x)=\max _{t \in\left[\tau_{1}, \tau_{4}\right]} x(t) \\
\alpha(x)=\min _{t \in\left[\tau_{2}, \tau_{3}\right]} x(t)
\end{array}\right.
$$

First, we shall show that the operator $S$ maps $\bar{P}\left(\gamma, w_{5}\right)$ into $\bar{P}\left(\gamma, w_{5}\right)$. Note that $\bar{P}\left(\gamma, w_{5}\right)=$ $\bar{C}\left(w_{5}\right)$. By (Q) and Lemma 3.3 (with $d=w_{5}$ ), we immediately have $S\left(\bar{C}\left(w_{5}\right)\right) \subseteq \bar{C}\left(w_{5}\right)$.

Next, to see that condition (a) of Theorem 2.2 is fulfilled, we note that

$$
\left\{x \in P\left(\gamma, \Theta, \alpha, w_{3}, w_{4}, w_{5}\right) \mid \alpha(x)>w_{3}\right\} \neq \emptyset
$$

since it has an element $x(t)=\frac{1}{2}\left(w_{3}+w_{4}\right)$. Let $x \in P\left(\gamma, \Theta, \alpha, w_{3}, w_{4}, w_{5}\right)$. Then by definition we have $\alpha(x) \geq w_{3}$ and $\Theta(x) \leq w_{4}$, which imply

$$
\begin{aligned}
& x(s) \in\left[w_{3}, w_{4}\right], \quad s \in\left[\tau_{2}, \tau_{3}\right] \quad \text { and } \\
& \int_{\tau_{2}}^{\frac{1}{2}} x(\tau) d \tau \in\left[w_{3}\left(\frac{1}{2}-\tau_{2}\right), w_{4}\left(\frac{1}{2}-\tau_{2}\right)\right] .
\end{aligned}
$$

Noting (3.7), (3.28), (C7) and (R), we find

$$
\begin{aligned}
\alpha(S x) & =\min _{t \in\left[\tau_{2}, \tau_{3}\right]} S x(t) \\
& \geq \min _{t \in\left[\tau_{2}, \tau_{3}\right]} \int_{0}^{1}\left|g_{m}(t, s)\right| \mu(s) f\left(\int_{0}^{s} x(\tau) d \tau, x(s)\right) d s \\
& \geq \min _{t \in\left[\tau_{2}, \tau_{3}\right]} \int_{\frac{1}{2}}^{\tau_{3}}\left|g_{m}(t, s)\right| \mu(s) f\left(\int_{\tau_{2}}^{\frac{1}{2}} x(\tau) d \tau, x(s)\right) d s \\
& >\min _{t \in\left[\tau_{2}, \tau_{3}\right]} \int_{\frac{1}{2}}^{\tau_{3}}\left|g_{m}(t, s)\right| \mu(s) d s \cdot \frac{w_{3}}{p_{1}} \\
& =p_{1} \cdot \frac{w_{3}}{p_{1}}=w_{3} .
\end{aligned}
$$

Hence, $\alpha(S x)>w_{3}$ for all $x \in P\left(\gamma, \Theta, \alpha, w_{3}, w_{4}, w_{5}\right)$. 
We shall now verify that condition (b) of Theorem 2.2 is satisfied. Let $w_{1}$ be such that $0<w_{1}<w_{2}$. Note that

$$
\left\{x \in Q\left(\gamma, \beta, \psi, w_{1}, w_{2}, w_{5}\right) \mid \beta(u)<w_{2}\right\} \neq \emptyset
$$

because it has an element $x(t)=\frac{1}{2}\left(w_{1}+w_{2}\right)$. Let $x \in Q\left(\gamma, \beta, \psi, w_{1}, w_{2}, w_{5}\right)$. Then we have $\beta(x) \leq w_{2}$ and $\gamma(x) \leq w_{5}$, i.e.,

$$
x(s) \in\left[0, w_{2}\right], \quad s \in\left[\tau_{1}, \tau_{4}\right] \quad \text { and } \quad x(s) \in\left[0, w_{5}\right], \quad s \in[0,1]
$$

which lead to the following:

$$
\begin{aligned}
\int_{0}^{s} x(\tau) d \tau & \leq w_{5}, \quad s \in\left[0, \tau_{1}\right] \cup\left[\tau_{4}, 1\right] \\
\int_{0}^{s} x(\tau) d \tau & \leq \int_{0}^{\tau_{4}} x(\tau) d \tau=\int_{0}^{\tau_{1}} x(\tau) d \tau+\int_{\tau_{1}}^{\tau_{4}} x(\tau) d \tau \\
& \leq \tau_{1} w_{5}+\left(\tau_{4}-\tau_{1}\right) w_{2}, \quad s \in\left[\tau_{1}, \tau_{4}\right] .
\end{aligned}
$$

Using (3.9), (3.29), (3.30), (C8), (P) and (Q) successively, we find

$$
\begin{aligned}
\beta(S x)= & \max _{t \in\left[\tau_{1}, \tau_{4}\right]} S x(t) \\
\leq & \frac{1}{\pi^{2 m-1}} \int_{0}^{1} v(s) f\left(\int_{0}^{s} x(\tau) d \tau, x(s)\right) \sin \pi s d s \\
= & \frac{1}{\pi^{2 m-1}}\left(\int_{0}^{\tau_{1}}+\int_{\tau_{1}}^{\tau_{4}}+\int_{\tau_{4}}^{1}\right) v(s) f\left(\int_{0}^{s} x(\tau) d \tau, x(s)\right) \sin \pi s d s \\
< & {\left[\frac{1}{\pi^{2 m-1}} \int_{0}^{\tau_{1}} v(s) \sin \pi s d s+\frac{1}{\pi^{2 m-1}} \int_{\tau_{4}}^{1} v(s) \sin \pi s d s\right] \frac{w_{5}}{q} } \\
& +\left[\frac{1}{\pi^{2 m-1}} \int_{\tau_{1}}^{\tau_{4}} v(s) \sin \pi s d s\right] \frac{1}{p_{2}}\left(w_{2}-\frac{w_{5} p_{3}}{q}\right) \\
= & p_{3} \cdot \frac{w_{5}}{q}+p_{2} \cdot \frac{1}{p_{2}}\left(w_{2}-\frac{w_{5} p_{3}}{q}\right)=w_{2} .
\end{aligned}
$$

Therefore, $\beta(S x)<w_{2}$ for all $x \in Q\left(\gamma, \beta, \psi, w_{1}, w_{2}, w_{5}\right)$.

Next, we shall show that condition (c) of Theorem 2.2 is met. Let $x \in C$. Clearly, we have

$$
\Theta(S x)=\max _{t \in\left[\tau_{1}, \tau_{4}\right]} S x(t) \leq\|S x\|
$$

Moreover, using the fact that $S$ maps $C$ into $C$, we find

$$
\alpha(S x)=\min _{t \in\left[\tau_{2}, \tau_{3}\right]} S x(t) \geq \min _{t \in[\delta, 1-\delta]} S x(t) \geq \frac{2 \delta \theta}{\pi}\|S x\| .
$$

Combining (3.31) and (3.32) yields

$$
\alpha(S x) \geq \frac{2 \delta \theta}{\pi} \Theta(S x), \quad x \in C .
$$


Now, let $x \in P\left(\gamma, \alpha, w_{3}, w_{5}\right)$ with $\Theta(S x)>w_{4}$. Then it follows from (3.33) and the inequality $\frac{\pi w_{3}}{2 \delta \theta} \leq w_{4}$ that

$$
\alpha(S x) \geq \frac{2 \delta \theta}{\pi} \Theta(S x)>\frac{2 \delta \theta}{\pi} w_{4} \geq \frac{2 \delta \theta}{\pi} \frac{\pi w_{3}}{2 \delta \theta}=w_{3} .
$$

Thus, $\alpha(S x)>w_{3}$ for all $x \in P\left(\gamma, \alpha, w_{3}, w_{5}\right)$ with $\Theta(S x)>w_{4}$.

Finally, we shall prove that condition (d) of Theorem 2.2 is fulfilled. Let $x \in Q\left(\gamma, \beta, w_{2}\right.$, $\left.w_{5}\right)$ with $\psi(S x)<w_{1}$. Then we have $\beta(x) \leq w_{2}$ and $\gamma(x) \leq w_{5}$ which give (3.29) and (3.30). As in proving condition (b), we get $\beta(S x)<w_{2}$. Hence, condition (d) of Theorem 2.2 is satisfied.

It now follows from Theorem 2.2 that the Lidstone boundary value problem (3.3) has (at least) three positive solutions $x_{1}, x_{2}, x_{3} \in \bar{P}\left(\gamma, w_{5}\right)=\bar{C}\left(w_{5}\right)$ satisfying (2.2). Furthermore, (2.2) reduces to (3.25) immediately. This completes the proof of conclusion (a).

Finally, as in the proof of Theorem 3.1, we see that (3.15) holds for the positive solutions $y_{i}, i=1,2,3$, of the complementary Lidstone boundary value problem (1.1). Moreover, noting that $x_{1}(t)<w_{2}$ for $t \in\left[\tau_{1}, \tau_{4}\right]$, we find for $t \in\left[\tau_{1}, \tau_{4}\right]$,

$$
\begin{aligned}
y_{1}(t) & =\int_{0}^{t} x_{1}(s) d s \leq \int_{0}^{\tau_{4}} x_{1}(s) d s=\int_{0}^{\tau_{1}} x_{1}(s) d s+\int_{\tau_{1}}^{\tau_{4}} x_{1}(s) d s \\
& <\tau_{1} \max _{s \in\left[0, \tau_{1}\right]} x_{1}(s)+\left(\tau_{4}-\tau_{1}\right) w_{2} .
\end{aligned}
$$

Next, noting $x_{2}(t)>w_{3}$ for $t \in\left[\tau_{2}, \tau_{3}\right]$, we get for $t \in\left[\tau_{2}, \tau_{3}\right]$,

$$
y_{2}(t)=\int_{0}^{t} x_{2}(s) d s>\int_{\tau_{2}}^{t} x_{2}(s) d s>w_{3}\left(t-\tau_{2}\right) .
$$

Lastly, using (3.15) and $\left\|x_{3}\right\| \geq \max _{t \in\left[\tau_{1}, \tau_{4}\right]} x_{3}(t)>w_{2}$, we find for $t \in[\delta, 1-\delta]$,

$$
y_{3}(t) \geq \frac{2 \delta \theta}{\pi}\left\|x_{3}\right\|(t-\delta)>\frac{2 \delta \theta}{\pi} w_{2}(t-\delta) .
$$

The proof of conclusion (b) is complete.

We shall now consider the special case of Theorem 3.2 when

$$
\tau_{1}=0, \quad \tau_{2}=\delta, \quad \tau_{3}=1-\delta \quad \text { and } \quad \tau_{4}=1 .
$$

Then, from definitions (3.11), we see that

$$
p_{1}=r, \quad p_{2}=q \text { and } \quad p_{3}=0 .
$$

In this case Theorem 3.2 yields the following corollary.

Corollary 3.1 Let $\delta \in\left(0, \frac{1}{2}\right)$ be fixed. Let $(\mathrm{C} 1)-(\mathrm{C} 4)$ hold, and assume

$(C 7)^{\prime}$ for each $t \in[\delta, 1-\delta]$, the function $\left|g_{m}(t, s)\right| \mu(s)>0$ on a subset of $\left[\frac{1}{2}, 1-\delta\right]$ of positive measure;

$(\mathrm{C} 8)^{\prime}$ the function $v(s) \sin \pi s>0$ on a subset of $[0,1]$ of positive measure. 
Suppose that there exist numbers $w_{i}, 2 \leq i \leq 5$, with

$$
0<w_{2}<w_{3}\left(\frac{1}{2}-\delta\right)<w_{3}<\frac{\pi w_{3}}{2 \delta \theta} \leq w_{4} \leq w_{5}
$$

such that the following hold:

(P) $f(u, v)<\frac{w_{2}}{q}$ for $u, v \in\left[0, w_{2}\right]$;

(Q) $f(u, v) \leq \frac{w_{5}}{q}$ for $u, v \in\left[0, w_{5}\right]$;

(R) $f(u, v)>\frac{w_{3}}{r}$ for $u \in\left[w_{3}\left(\frac{1}{2}-\delta\right), w_{4}\left(\frac{1}{2}-\delta\right)\right]$ and $v \in\left[w_{3}, w_{4}\right]$.

Then we have the following conclusions:

(a) The Lidstone boundary value problem (3.3) has (at least) three positive solutions $x_{1}, x_{2}, x_{3} \in \bar{C}\left(w_{5}\right)$ (where $C$ is defined in (3.8)) such that

$$
\left\{\begin{array}{l}
\left\|x_{1}\right\|<w_{2} ; \\
x_{2}(t)>w_{3}, \quad t \in[\delta, 1-\delta] \\
\left\|x_{3}\right\|>w_{2} \quad \text { and } \min _{t \in[\delta, 1-\delta]} x_{3}(t)<w_{3} .
\end{array}\right.
$$

(b) The complementary Lidstone boundary value problem (1.1) has (at least) three positive solutions $y_{1}, y_{2}, y_{3}$ such that (3.15) holds for $i=1,2,3$. We further have

$$
\left\{\begin{array}{l}
\left\|y_{1}\right\|<w_{2} \\
y_{2}(t)>w_{3}(t-\delta), \quad t \in[\delta, 1-\delta] \\
y_{3}(t)>\frac{2 \delta \theta}{\pi} w_{2}(t-\delta), \quad t \in[\delta, 1-\delta]
\end{array}\right.
$$

Remark 3.1 Corollary 3.1 is actually Theorem 3.1. Since Corollary 3.1 is a special case of Theorem 3.2, this shows that Theorem 3.2 is more general than Theorem 3.1.

The next theorem illustrates another application of Theorem 2.2. Compared to the conditions in Theorem 3.2, here the numbers $w_{1}, \tau_{1}$ and $\tau_{4}$ have different ranges and condition (P) is also different. Note that in the proof of Theorem 3.3 the functionals $\psi$ and $\Theta$ are chosen differently from those in Theorem 3.2.

Theorem 3.3 Let $\delta \in\left(0, \frac{1}{2}\right)$ be fixed. Let (C1)-(C4) hold. Assume that there exist numbers $\tau_{j}, 1 \leq j \leq 4$, with

$$
\delta \leq \tau_{1} \leq \tau_{2}<\frac{1}{2}<\tau_{3} \leq \tau_{4} \leq 1-\delta
$$

such that (C7) and (C8) hold. Suppose that there exist numbers $w_{i}, 1 \leq i \leq 5$, with

$$
\left\{\begin{array}{l}
0<w_{1} \leq \frac{2 \delta \theta w_{2}}{\pi}<w_{2}<w_{3}<\frac{\pi w_{3}}{2 \delta \theta} \leq w_{4} \leq w_{5}<\frac{q w_{2}}{p_{3}} \\
\tau_{1} w_{5}+\left(\tau_{4}-\tau_{1}\right) w_{2}<w_{3}\left(\frac{1}{2}-\tau_{2}\right)
\end{array}\right.
$$

such that the following hold:

(P) $f(u, v)<\frac{1}{p_{2}}\left(w_{2}-\frac{w_{5} p_{3}}{q}\right)$ for $u \in\left[0, \tau_{1} w_{5}+\left(\tau_{4}-\tau_{1}\right) w_{2}\right]$ and $v \in\left[w_{1}, w_{2}\right]$;

(Q) $f(u, v) \leq \frac{w_{5}}{q}$ for $u, v \in\left[0, w_{5}\right]$;

(R) $f(u, v)>\frac{w_{3}}{p_{1}}$ for $u \in\left[w_{3}\left(\frac{1}{2}-\tau_{2}\right), w_{4}\left(\frac{1}{2}-\tau_{2}\right)\right]$ and $v \in\left[w_{3}, w_{4}\right]$.

Then we have conclusions (a) and (b) of Theorem 3.2. 
Proof To apply Theorem 2.2, we shall define the following functionals on the cone $C$ (see (3.8)):

$$
\left\{\begin{array}{l}
\gamma(x)=\|x\|, \\
\psi(x)=\min _{t \in\left[\tau_{1}, \tau_{4}\right]} x(t), \\
\beta(x)=\max _{t \in\left[\tau_{1}, \tau_{4}\right]} x(t), \\
\alpha(u)=\min _{t \in\left[\tau_{2}, \tau_{3}\right]} x(t), \\
\Theta(u)=\max _{t \in\left[\tau_{2}, \tau_{3}\right]} x(t) .
\end{array}\right.
$$

As in the proof of Theorem 3.2, using (Q) and Lemma 3.3 we can show that $S: \bar{P}(\gamma$, $\left.w_{5}\right) \rightarrow \bar{P}\left(\gamma, w_{5}\right)$.

Next, to see that condition (a) of Theorem 2.2 is fulfilled, we use (R) and a similar argument as in the proof of Theorem 3.2.

We shall now prove that condition (b) of Theorem 2.2 is satisfied. Note that

$$
x(t)=\frac{1}{2}\left(w_{1}+w_{2}\right) \in\left\{x \in Q\left(\gamma, \beta, \psi, w_{1}, w_{2}, w_{5}\right) \mid \beta(u)<w_{2}\right\} \neq \emptyset .
$$

Let $x \in Q\left(\gamma, \beta, \psi, w_{1}, w_{2}, w_{5}\right)$. Then we have $\psi(x) \geq w_{1}, \beta(x) \leq w_{2}$ and $\gamma(x) \leq w_{5}$ which imply

$$
x(s) \in\left[w_{1}, w_{2}\right], \quad s \in\left[\tau_{1}, \tau_{4}\right] \quad \text { and } \quad x(s) \in\left[0, w_{5}\right], \quad s \in[0,1]
$$

and also (3.30). In view of (3.9), (3.38), (3.30), (C8), (P) and (Q), we obtain, as in the proof of Theorem 3.2, that $\beta(S x)<w_{2}$. Therefore, condition (b) of Theorem 2.2 is fulfilled.

Next, using a similar argument as in the proof of Theorem 3.2, we see that condition (c) of Theorem 2.2 is met.

Finally, we shall verify that condition (d) of Theorem 2.2 is fulfilled. Let $x \in C$. It is clear that

$$
\beta(S x)=\max _{t \in\left[\tau_{1}, \tau_{4}\right]} S x(t) \leq\|S x\| .
$$

Noting that $S$ maps $C$ into $C$, we find

$$
\psi(S x)=\min _{t \in\left[\tau_{1}, \tau_{4}\right]} S x(t) \geq \min _{t \in[\delta, 1-\delta]} S x(t) \geq \frac{2 \delta \theta}{\pi}\|S x\| .
$$

A combination of (3.39) and (3.40) gives

$$
\psi(S x) \geq \frac{2 \delta \theta}{\pi} \beta(S x), \quad x \in C .
$$

Let $x \in Q\left(\gamma, \beta, w_{2}, w_{5}\right)$ with $\psi(S x)<w_{1}$. Then (3.41) and the inequality $w_{1} \leq \frac{2 \delta \theta w_{2}}{\pi}$ lead to

$$
\beta(S x) \leq \frac{\pi}{2 \delta \theta} \psi(S x)<\frac{\pi}{2 \delta \theta} w_{1} \leq \frac{\pi}{2 \delta \theta} \frac{2 \delta \theta}{\pi} w_{2}=w_{2} .
$$

Thus, $\beta(S x)<w_{2}$ for all $x \in Q\left(\gamma, \beta, w_{2}, w_{5}\right)$ with $\psi(S x)<w_{1}$.

Conclusion (a) now follows from Theorem 2.2 immediately, while conclusion (b) is similarly obtained as in Theorem 3.2 . 


\section{Examples}

In this section, we shall present examples to illustrate the usefulness as well as to compare the generality of the results obtained in Section 3.

Example 4.1 Consider the complementary Lidstone boundary value problem (1.1) with $m=3$ and the nonlinear term $F$ given by

$$
\begin{aligned}
F(t, u, v) & =f(u, v) \\
& = \begin{cases}\frac{w_{1}}{2 q}, & (u, v) \in\left[0, w_{1}\right] \times\left[0, w_{1}\right] \equiv A_{1}, \\
\frac{1}{2}\left(\frac{d}{q}+\frac{w_{2}}{r}\right), & (u, v) \in\left[w_{2}\left(\frac{1}{2}-\delta\right), \infty\right) \times\left[w_{2}, \infty\right) \equiv A_{2}, \\
\phi(u, v), & (u, v) \in \mathbb{R}^{2} \backslash\left(A_{1} \cup A_{2}\right),\end{cases}
\end{aligned}
$$

where $\phi(u, v)$ is continuous in each argument and satisfies

$$
\left\{\begin{array}{l}
\phi(0, v)=\phi\left(w_{1}, v\right)=\phi(u, 0)=\phi\left(u, w_{1}\right)=\frac{w_{1}}{2 q}, \quad u, v \in\left[0, w_{1}\right] \\
\phi\left(w_{2}\left(\frac{1}{2}-\delta\right), v\right)=\phi\left(u, w_{2}\right)=\frac{1}{2}\left(\frac{d}{q}+\frac{w_{2}}{r}\right), \quad u \in\left[w_{2}\left(\frac{1}{2}-\delta\right), \infty\right), v \in\left[w_{2}, \infty\right) \\
0 \leq \phi(u, v) \leq \frac{1}{2}\left(\frac{d}{q}+\frac{w_{2}}{r}\right), \quad(u, v) \in \mathbb{R}^{2} \backslash\left(A_{1} \cup A_{2}\right) .
\end{array}\right.
$$

Here, $\delta=\frac{1}{4}$ is fixed and the $w_{i}$ 's and $d$ are in the context of Theorem 3.1 satisfying

$$
0<w_{1}<w_{2}\left(\frac{1}{2}-\delta\right)<w_{2}<\frac{\pi w_{2}}{2 \delta \theta} \leq w_{3} \leq d \quad \text { and } \quad d>\frac{q w_{2}}{r} .
$$

Let the functions $\mu=v \equiv 1$ (which implies $\theta=1$ ). Then it is clear that (C1)-(C6) are fulfilled. Moreover, by direct computation we get $q=2.080 \times 10^{-3}$, and on using Lemma 2.2 we find

$$
r=\min _{t \in[\delta, 1-\delta]} \int_{\frac{1}{2}}^{1-\delta}\left|g_{m}(t, s)\right| \mu(s) d s \geq \frac{2 \delta}{\pi^{2 m}} \int_{\frac{1}{2}}^{1-\delta} \sin \pi s d s=1.171 \times 10^{-4} .
$$

For convenience, we take $r=1.171 \times 10^{-4}$ although this will lead to more stringent conditions.

Hence, (4.3) reduces to

$$
0<w_{1}<0.25 w_{2}<w_{2}<6.283 w_{2} \leq w_{3} \leq d \quad \text { and } \quad d>17.77 w_{2}
$$

and clearly we can easily find numbers $w_{i}$ 's and $d$ that satisfy (4.4).

We shall check the conditions of Theorem 3.1. First, condition $(\mathrm{P})$ is obviously satisfied. Next, from (4.3) we have $\frac{w_{2}}{r}<\frac{d}{q}$, therefore for $(u, v) \in[0, d] \times[0, d]$ it follows that

$$
f(u, v) \leq \frac{1}{2}\left(\frac{d}{q}+\frac{w_{2}}{r}\right)<\frac{1}{2}\left(\frac{d}{q}+\frac{d}{q}\right)=\frac{d}{q} .
$$

Hence, condition $(\mathrm{Q} 2)$ is met. Finally, $(\mathrm{R})$ is satisfied since for $(u, v) \in\left[w_{2}\left(\frac{1}{2}-\delta\right), w_{3}\left(\frac{1}{2}-\right.\right.$ $\delta)] \times\left[w_{2}, w_{3}\right]$, we have

$$
f(u, v)=\frac{1}{2}\left(\frac{d}{q}+\frac{w_{2}}{r}\right)>\frac{1}{2}\left(\frac{w_{2}}{r}+\frac{w_{2}}{r}\right)=\frac{w_{2}}{r} .
$$


By Theorem 3.1 (conclusion (b)), the boundary value problem (1.1) with $m=3, \delta=\frac{1}{4}$, (4.1) and (4.2) has (at least) three positive solutions $y_{1}, y_{2}, y_{3}$ such that (from (3.16))

$$
\left\{\begin{array}{l}
\left\|y_{1}\right\|<w_{1} \\
y_{2}(t)>w_{2}\left(t-\frac{1}{4}\right), \quad t \in\left[\frac{1}{4}, \frac{3}{4}\right] \\
y_{3}(t)>\frac{1}{2 \pi} w_{1}\left(t-\frac{1}{4}\right), \quad t \in\left[\frac{1}{4}, \frac{3}{4}\right]
\end{array}\right.
$$

where $w_{i}$ 's satisfy (4.4).

Example 4.2 Consider the complementary Lidstone boundary value problem (1.1) with $m=3$ and the nonlinear term $F$ given by

$$
\begin{aligned}
F(t, u, v) & =f(u, v) \\
& = \begin{cases}\frac{1}{2 p_{2}}\left(w_{2}-\frac{w_{5} p_{3}}{q}\right), & (u, v) \in\left[0, \tau_{1} w_{5}+\left(\tau_{4}-\tau_{1}\right) w_{2}\right] \times\left[0, w_{2}\right] \equiv A_{3}, \\
\frac{1}{2}\left(\frac{w_{3}}{r}+\frac{w_{3}}{p_{1}}\right), & (u, v) \in\left[w_{3}\left(\frac{1}{2}-\tau_{2}\right), \infty\right) \times\left[w_{3}, \infty\right) \equiv A_{4}, \\
\kappa(u, v), & (u, v) \in \mathbb{R}^{2} \backslash\left(A_{3} \cup A_{4}\right),\end{cases}
\end{aligned}
$$

where $\kappa(u, v)$ is continuous in each argument and satisfies

$$
\left\{\begin{array}{c}
\kappa(0, v)=\kappa\left(\tau_{1} w_{5}+\left(\tau_{4}-\tau_{1}\right) w_{2}, v\right)=\kappa(u, 0)=\kappa\left(u, w_{2}\right)=\frac{1}{2 p_{2}}\left(w_{2}-\frac{w_{5} p_{3}}{q}\right), \\
u \in\left[0, \tau_{1} w_{5}+\left(\tau_{4}-\tau_{1}\right) w_{2}\right], v \in\left[0, w_{2}\right] ; \\
\kappa\left(w_{3}\left(\frac{1}{2}-\tau_{2}\right), v\right)=\kappa\left(u, w_{3}\right)=\frac{1}{2}\left(\frac{w_{3}}{r}+\frac{w_{3}}{p_{1}}\right), \quad u \in\left[w_{3}\left(\frac{1}{2}-\tau_{2}\right), \infty\right), v \in\left[w_{3}, \infty\right) ; \\
0 \leq \kappa(u, v) \leq \frac{1}{2}\left(\frac{w_{3}}{r}+\frac{w_{3}}{p_{1}}\right), \quad(u, v) \in \mathbb{R}^{2} \backslash\left(A_{3} \cup A_{4}\right) .
\end{array}\right.
$$

Here, we fix

$$
\delta=0.05, \quad \tau_{1}=0, \quad \tau_{2}=0.1, \quad \tau_{3}=\tau_{4}=1-\delta=0.95
$$

and the $w_{i}$ 's are in the context of Theorem 3.2 satisfying

$$
\left\{\begin{array}{l}
0<w_{2}<w_{3}<\frac{\pi w_{3}}{2 \delta \theta} \leq w_{4} \leq w_{5}<\frac{q w_{2}}{p_{3}} \\
\tau_{1} w_{5}+\left(\tau_{4}-\tau_{1}\right) w_{2}<w_{3}\left(\frac{1}{2}-\tau_{2}\right) \quad \text { and } \quad w_{5}>\frac{q w_{3}}{r} .
\end{array}\right.
$$

Let the functions $\mu=v \equiv 1$ (which implies $\theta=1$ ). Then it is clear that (C1)-(C4), (C7) and (C8) are fulfilled. Moreover, by direct computation we have

$$
q=2.080 \times 10^{-3}, \quad p_{2}=2.068 \times 10^{-3}, \quad p_{3}=1.281 \times 10^{-5} .
$$

By using Lemma 2.2, we get

$$
\begin{aligned}
p_{1} & =\min _{t \in[0.1,0.95]} \int_{\frac{1}{2}}^{0.95}\left|g_{m}(t, s)\right| \mu(s) d s \\
> & r=\min _{t \in[0.05,0.95]} \int_{\frac{1}{2}}^{0.95}\left|g_{m}(t, s)\right| \mu(s) d s \\
& \geq \frac{2 \delta}{\pi^{2 m}} \int_{\frac{1}{2}}^{0.95} \sin \pi s d s \\
& =3.270 \times 10^{-5} .
\end{aligned}
$$


For convenience, we take $r=3.270 \times 10^{-5}$ although this will lead to more stringent conditions. Hence, (4.9) reduces to

$$
\left\{\begin{array}{l}
0<w_{2}<w_{3}<31.42 w_{3} \leq w_{4} \leq w_{5}<162.4 w_{2} \\
0.95 w_{2}<0.4 w_{3} \text { and } w_{5}>63.61 w_{3}
\end{array}\right.
$$

or equivalently (combining the first two inequalities)

$$
\left\{\begin{array}{l}
0<w_{2}<w_{3}<31.42 w_{3} \leq w_{4} \leq w_{5}<162.4 w_{2}<68.38 w_{3} \quad \text { and } \\
w_{5}>63.61 w_{3} .
\end{array}\right.
$$

It is clear that we can easily find numbers $w_{i}$ 's that fulfill (4.10).

We shall check the conditions of Theorem 3.2. First, condition (P) is obviously satisfied. Next, since

$$
r<p_{1}<p_{2} \quad \text { and } \quad \frac{w_{3}}{r}<\frac{w_{5}}{q} \quad\left(\text { i.e., } w_{5}>63.61 w_{3}\right),
$$

we find for $(u, v) \in\left[0, w_{5}\right] \times\left[0, w_{5}\right]$,

$$
f(u, v) \leq \frac{1}{2}\left(\frac{w_{3}}{r}+\frac{w_{3}}{p_{1}}\right)<\frac{1}{2}\left(\frac{w_{3}}{r}+\frac{w_{3}}{r}\right)=\frac{w_{3}}{r}<\frac{w_{5}}{q} .
$$

Hence, condition $(\mathrm{Q})$ is met. Finally, $(\mathrm{R})$ is satisfied since for $(u, v) \in\left[w_{3}\left(\frac{1}{2}-\tau_{2}\right), w_{4}\left(\frac{1}{2}-\right.\right.$ $\left.\left.\tau_{2}\right)\right] \times\left[w_{3}, w_{4}\right]$, using $(4.11)$ we get

$$
f(u, v)=\frac{1}{2}\left(\frac{w_{3}}{r}+\frac{w_{3}}{p_{1}}\right)>\frac{1}{2}\left(\frac{w_{3}}{p_{1}}+\frac{w_{3}}{p_{1}}\right)=\frac{w_{3}}{p_{1}} .
$$

By Theorem 3.2 (conclusion (b)), the boundary value problem (1.1) with $m=3$, (4.6), (4.7) and (4.8) has (at least) three positive solutions $y_{1}, y_{2}, y_{3}$ such that (from (3.26))

$$
\left\{\begin{array}{l}
y_{1}(t)<0.95 w_{2}, \quad t \in[0,0.95] \\
y_{2}(t)>w_{3}(t-0.1), \quad t \in[0.1,0.95] \\
y_{3}(t)>\frac{1}{10 \pi} w_{2}(t-0.05), \quad t \in[0.05,0.95]
\end{array}\right.
$$

where $w_{i}^{\prime}$ 's satisfy (4.10).

Remark 4.1 In Example 4.2, we see that for $(u, v) \in\left[w_{3}\left(\frac{1}{2}-\tau_{2}\right), w_{4}\left(\frac{1}{2}-\tau_{2}\right)\right] \times\left[w_{3}, w_{4}\right]$,

$$
f(u, v)=\frac{1}{2}\left(\frac{w_{3}}{r}+\frac{w_{3}}{p_{1}}\right)<\frac{1}{2}\left(\frac{w_{3}}{r}+\frac{w_{3}}{r}\right)=\frac{w_{3}}{r} .
$$

Thus, condition (R) of Corollary 3.1 is not satisfied and so Corollary 3.1 cannot be used to establish the existence of triple positive solutions in Example 4.2. Recalling that Corollary 3.1 is actually Theorem 3.1, this illustrates the case when Theorem 3.2 is applicable but not Theorem 3.1. Hence, this example shows that Theorem 3.2 is indeed more general than Theorem 3.1 . 


\section{Competing interests}

The author declares that she has no competing interests.

\section{Received: 25 June 2013 Accepted: 15 August 2013 Published: 20 May 2014}

\section{References}

1. Costabile, FA, Dell'Accio, F, Luceri, R: Explicit polynomial expansions of regular real functions by means of even order Bernoulli polynomials and boundary values. J. Comput. Appl. Math. 175, 77-99 (2005)

2. Agarwal, RP, Pinelas, S, Wong, PJY: Complementary Lidstone interpolation and boundary value problems. J. Inequal. Appl. 2009, 624631 (2009)

3. Agarwal, RP, Wong, PJY: Piecewise complementary Lidstone interpolation and error inequalities. J. Comput. Appl. Math. 234, 2543-2561 (2010)

4. Agarwal, RP, Wong, PJY: Lidstone polynomials and boundary value problems. Comput. Math. Appl. 17, 1397-1421 (1989)

5. Agarwal, RP, Wong, PJY: Error Inequalities in Polynomial Interpolation and Their Applications. Kluwer Academic, Dordrecht (1993)

6. Davis, PJ: Interpolation and Approximation. Blaisdell, Boston (1961)

7. Varma, AK, Howell, G: Best error bounds for derivatives in two point Birkhoff interpolation problem. J. Approx. Theory $38,258-268(1983)$

8. Lidstone, GJ: Notes on the extension of Aitken's theorem (for polynomial interpolation) to the Everett types. Proc Edinb. Math. Soc. 2, 16-19 (1929)

9. Boas, RP: A note on functions of exponential type. Bull. Am. Math. Soc. 47, 750-754 (1941)

10. Boas, RP: Representation of functions by Lidstone series. Duke Math. J. 10, $239-245$ (1943)

11. Poritsky, H: On certain polynomial and other approximations to analytic functions. Trans. Am. Math. Soc. 34, 274-331 (1932)

12. Schoenberg, IJ: On certain two-point expansions of integral functions of exponential type. Bull. Am. Math. Soc. 42, 284-288 (1936)

13. Whittaker, JM: On Lidstone's series and two-point expansions of analytic functions. Proc. Lond. Math. Soc. 36, 451-469 (1933-1934)

14. Whittaker, JM: Interpolatory Function Theory. Cambridge University Press, Cambridge (1935)

15. Widder, DV: Functions whose even derivatives have a prescribed sign. Proc. Natl. Acad. Sci. USA 26, 657-659 (1940)

16. Widder, DV: Completely convex functions and Lidstone series. Trans. Am. Math. Soc. 51, $387-398$ (1942)

17. Agarwal, RP, Wong, PJY: Explicit error bounds for the derivatives of piecewise-Lidstone interpolation. J. Comput. Appl. Math. 58, 67-81 (1995)

18. Agarwal, RP, Wong, PJY: Error bounds for the derivatives of Lidstone interpolation and applications. In: Govil, NK Mohapatra, RN, Nashed, Z, Sharma, A, Szabados, J (eds.) Approximation Theory: In Memory of A.K. Varma, pp. 1-41. Dekker, New York (1998)

19. Costabile, FA, Dell'Accio, F: Lidstone approximation on the triangle. Appl. Numer. Math. 52, 339-361 (2005)

20. Costabile, FA, Serpe, A: An algebraic approach to Lidstone polynomials. Appl. Math. Lett. 20, 387-390 (2007)

21. Wong, PJY: On Lidstone splines and some of their applications. Neural Parallel Sci. Comput. 1, 472-475 (1995)

22. Wong, PJY, Agarwal, RP: Sharp error bounds for the derivatives of Lidstone-spline interpolation. Comput. Math. Appl. 28(9), 23-53 (1994)

23. Wong, PJY, Agarwal, RP: Sharp error bounds for the derivatives of Lidstone-spline interpolation II. Comput. Math Appl. 31(3), 61-90 (1996)

24. Agarwal, RP, Akrivis, G: Boundary value problems occurring in plate deflection theory. J. Comput. Appl. Math. 8 145-154 (1982)

25. Agarwal, RP, O'Regan, D, Wong, PJY: Positive Solutions of Differential, Difference and Integral Equations. Kluwer Academic, Dordrecht (1999)

26. Agarwal, RP, Wong, PJY: Quasilinearization and approximate quasilinearization for Lidstone boundary value problems. Int. J. Comput. Math. 42, 99-116 (1992)

27. Baldwin, P: Asymptotic estimates of the eigenvalues of a sixth-order boundary-value problem obtained by using global phase-integral method. Philos. Trans. R. Soc. Lond. Ser. A 322, 281-305 (1987)

28. Baldwin, P: A localized instability in a Bénard layer. Appl. Anal. 24, 117-156 (1987)

29. Boutayeb, A, Twizell, EH: Finite-difference methods for twelfth-order boundary value problems. J. Comput. Appl. Math. 35, 133-138 (1991)

30. Davis, JM, Eloe, PW, Henderson, J: Triple positive solutions and dependence on higher order derivatives. J. Math. Anal. Appl. 237, 710-720 (1999)

31. Davis, JM, Henderson, J, Wong, PJY: General Lidstone problems: multiplicity and symmetry of solutions. J. Math. Anal. Appl. 251, 527-548 (2000)

32. Eloe, PW, Henderson, J, Thompson, HB: Extremal points for impulsive Lidstone boundary value problems. Math. Comput. Model. 32, 687-698 (2000)

33. Forster, $P$ : Existenzaussagen und Fehlerabschätzungen bei gewissen nichtlinearen Randwertaufgaben mit gewöhnlichen Differentialgleichungen. Numer. Math. 10, 410-422 (1967)

34. Guo, Y, Ge, W: Twin positive symmetric solutions for Lidstone boundary value problems. Taiwan. J. Math. 8, 271-283 (2004)

35. Ma, Y: Existence of positive solutions of Lidstone boundary value problems. J. Math. Anal. Appl. 314, 97-108 (2006)

36. Twizell, EH, Boutayeb, A: Numerical methods for the solution of special and general sixth-order boundary value problems, with applications to Bénard layer eigenvalue problems. Proc. R. Soc. Lond. Ser. A 431, 433-450 (1990)

37. Yao, Q: On the positive solutions of Lidstone boundary value problems. Appl. Math. Comput. 137, 477-485 (2003)

38. Agarwal, RP, Wong, PJY: Eigenvalues of complementary Lidstone boundary value problems. Bound. Value Probl. 2012, 49 (2012)

39. Agarwal, RP, Wong, PJY: Positive solutions of complementary Lidstone boundary value problems. Electron. J. Qual. Theory Differ. Equ. 2012, 60 (2012) 
40. Leggett, RW, Williams, LR: Multiple positive fixed points of nonlinear operators on ordered Banach spaces. Indiana Univ. Math. J. 28, 673-688 (1979)

41. Avery, Rl: A generalization of the Leggett-Williams fixed point theorem. Math. Sci. Res. Hot-Line 2, 9-14 (1998)

42. O'Regan, D, Meehan, M: Existence Theory for Nonlinear Integral and Integrodifferential Equations. Kluwer Academic, Dordrecht (1998)

10.1186/1687-2770-2014-125

Cite this article as: Wong: Triple solutions of complementary Lidstone boundary value problems via fixed point theorems. Boundary Value Problems 2014, 2014:125

Submit your manuscript to a SpringerOpen ${ }^{\circ}$ journal and benefit from:

- Convenient online submission

- Rigorous peer review

- Immediate publication on acceptance

- Open access: articles freely available online

- High visibility within the field

- Retaining the copyright to your article

Submit your next manuscript at $>$ springeropen.com 